Objectives: 1) To assess the future risk of newly recorded MI and CVA events among incident cases of AS compared to non-AS controls from the general population by utilizing physician billing, medication, and hospitalization data that covers the entire province of British Columbia (BC), Canada.

Methods: Our data includes all outpatient visits and hospitalizations (1990-2012) and all dispensed medications (1996-2012) for all BC residents. We conducted a retrospective matched cohort study of all patients $>18$ years of age satisfying the following criteria: 1) two ICD-9 or 10 codes (720.0 or M45) for AS at least two months apart and within a 2-year period by any physician or hospitalization; 2) all AS cases had at least a 7-year run-in period before the 1st ICD code for AS in order to consider the case as incident. Each AS patient was matched with up to 10 controls by birth year, sex, and entry cohort time. The outcomes were a newly recorded MI (ICD-9-CM: 410 or ICD-10-CM: I21) or CVA (ICD-9 codes: 433-434, ICD-10 codes: 163-166) event from hospital or death certificates. We estimated relative risks (RRs), adjusting for age, sex, and entry cohort time as well as multivariable models adjusting for confounders including glucocorticoids and non-steroidal anti-inflammatory drugs using a Cox proportional hazard model.

Results: 7,190 individuals with newly diagnosed AS were identified $(48.7 \%$ female, mean age of $45.8 \mathrm{yrs}$ ). 7,148 and 7,107 were free of previous CVA/MI, respectively. 80 developed CVA (incidence rate $=1.8$ per 1000 patient years) and 115 had Ml (incidence rate $=2.6$ per 1,000 patient years) (Table 1). The age-, sex-, and entry-time-matched RR for CVA was $1.60(95 \% \mathrm{Cl}, 1.25-2.03)$ and $\mathrm{MI}$ was $1.52(95 \% \mathrm{Cl}, 1.24-1.85)$. When adjusted for cardiovascular risk factors (obesity, angina, COPD, hospitalizations in year before index date, Charlson's comorbidity index, oral glucocorticoids, cardiovascular drugs, anti-diabetic medication, HRT, contraceptives, fibrates, statins, NSAIDs, and Cox-2 inhibitors), the estimated RR was 1.34 (1.04-1.73) for CVA and $1.21(0.98-1.49)$ for MI.

\begin{tabular}{|c|c|c|}
\hline & AS $(N=7,148)$ & Non-AS $(\mathrm{N}=71,489)$ \\
\hline CVA events, $n$ & 80 & 492 \\
\hline Incidence Rate of CVA/1000 Person-Years & 1.81 & 1.13 \\
\hline Incidence Rate Ratio of CVA (95\% Cl) & $1.60(1.25-2.03)$ & 1.0 \\
\hline \multirow[t]{2}{*}{ Multivariable RR of CVA $(95 \% \mathrm{Cl})$} & $1.34(1.04-1.73)$ & 1.0 \\
\hline & AS $(\mathrm{N}=7,107)$ & Non-AS $(\mathrm{N}=71,033)$ \\
\hline Ml events, $\mathrm{n}$ & 115 & 748 \\
\hline Incidence Rate of $\mathrm{Ml} / 1000$ Person-Years & 2.62 & 1.73 \\
\hline Incidence Rate Ratio of MI (95\% Cl) & $1.52(1.24-1.85)$ & 1.0 \\
\hline Multivariable RR of $\mathrm{MI}(95 \% \mathrm{Cl})$ & $1.21(0.98-1.49)$ & 1.0 \\
\hline
\end{tabular}

Conclusions: This large population-based study demonstrates an increased risk of CVA, but not for MI. These findings support that increased monitoring for this potentially fatal outcome and its modifiable risk factors is warranted for AS patients.

Disclosure of Interest: None declared

DOI: 10.1136/annrheumdis-2017-eular.6544

\section{SAT0385 SIMILARITIES AND DIFFERENCES BETWEEN PATIENTS FULFILLING NON-RADIOGRAPHIC AXIAL SPONDYLOARTHRITIS AND UNDIFFERENTIATED SPONDYLOARTHRITIS CRITERIA: RESULTS FROM THE ESPERANZA COHORT}

A. Juan Mas ${ }^{1}$, X. Juanola Roura ${ }^{2}$, E. de Miguel Mendieta ${ }^{3}$, E. Collantes Estévez $^{4}$, J.C. Quevedo Abeledo ${ }^{5}$, E. Alonso Blanco-Morales ${ }^{6}$,

V. Navarro-Compán ${ }^{3}$ on behalf of ESPeranza working group. ${ }^{1}$ Rheumatology, Hospital Son Llàtzer, Palma de Mallorca; ${ }^{2}$ Hospital Bellvitge, Barcelona;

${ }^{3}$ Hospital la Paz, IdiPaz, Madrid; ${ }^{4}$ Hospital Reina Sofía, Córdoba; ${ }^{5}$ Hospital Gran Canaria Dr. Negrín, Gran Canaria; ${ }^{6}$ Hospital Juan Canalejo, A Coruña, Spain

Background: Patients with spondyloarthritis $(\mathrm{SpA})$ were classified in five subgroups: ankylosing spondylitis (AS), psoriatic arthritis, arthritis associated with inflammatory bowel disease, reactive arthritis and undifferentiated SpA (uSpA). ASAS criteria classify patients in peripheral SpA and axial SpA (axSpA), being the latest classified in two groups: classical AS and non-radiographic axSpA (nr-axSpa). Whether or not patients with nr-axSpA represent the same group of patients that used to be classified as USpA remains unclear.

Objectives: To evaluate the similarities and differences between patients with predominant axial disease classified currently as $\mathrm{nr}$-axSpA versus those traditionally classified as USpA.

Methods: Baseline data from the ESPeranza program (a multicenter national initiative to early diagnose SpA between 2008 and 2011) was used. Inclusion criteria for this program were: age $<45$ years and inflammatory back pain plus $\geq 1$ SpA features with symptoms duration between 3 and 24 months. Demographic, clinic, laboratory and image results were compared between two groups: 182 patients with nr-axSpA and 166 patients classified as USpA. In order to get a deeper knowledge of the differences between nr-axSpA and uSpA, we also compared: i) 88 patients only classified as nr-axSpA, ii) 72 patients only classified as uSpA; iii) 94 patients fulfilling both criteria. Student-t test for continuous variables and Pearson Chi-square test for categorical variables were used. Results: Compared to patients classified as uSpA patients with nr-axSpA were younger, had HLA-B27 positive more frequently and higher values of CRP. On the other hand, they had history of SpA less frequently and lower values for BASDAI, BASFI and ASQoL (table). No differences were observed for gender, work incapacity, dactilitis, enthesitis, Pt's and Phy's VAS, BASMI and BASRI.

Table 1. Results are presented in mean \pm standard deviation for continuous variables and $\mathrm{n}(\%)$ for categorical variables

\begin{tabular}{lcccc}
\hline & $\begin{array}{c}\text { Both, } \\
\text { nr-axSpA \& undifferentiated SpA } \\
\mathrm{N}(\%)=94\end{array}$ & $\begin{array}{c}\text { Only } \\
\mathrm{nr}-\mathrm{axSpA} \\
\mathrm{N}(\%)=88\end{array}$ & $\begin{array}{c}\text { Only undifferentiated } \\
\text { SpA }\end{array}$ & $\mathrm{p}$ value* \\
$\mathrm{N}(\%)=72$ & \\
\hline Age (years) & $30.9 \pm 7.3$ & $32.2 \pm 6.9$ & $35.2 \pm 6.9$ & $<\mathbf{0 . 0 1}$ \\
Male & $61(64.9)$ & $50(56.8)$ & $34(47.2)$ & 0.2 \\
Family history & $48(51.1)$ & $21(23.9)$ & $30(41.7)$ & $<0.05$ \\
HLA-B27 & $81(86.2)$ & $65(73.9)$ & $6(8.3)$ & $<0.001$ \\
Enthesitis & $29(30.9)$ & $18(20.5)$ & $22(30.6)$ & 0.1 \\
BASDAI & $3.7 \pm 2.3$ & $3.8 \pm 2.1$ & $4.7 \pm 2.3$ & $<0.01$ \\
BASFI & $2.2 \pm 2.2$ & $2.1 \pm 2.1$ & $2.9 \pm 2.5$ & $<\mathbf{0 . 0 5}$ \\
ASQoL & $5.9 \pm 4.7$ & $5.2 \pm 4.5$ & $7.4 \pm 5.2$ & $\mathbf{0 . 0 1}$ \\
CRP (mg/L) & $10.7 \pm 14.3$ & $9.8 \pm 16.5$ & $5.1 \pm 9.1$ & $<\mathbf{0 . 0 5}$ \\
\hline
\end{tabular}

${ }^{*} \mathrm{p}$ value for differences between $\mathrm{nr}-\mathrm{axSpA}$ and undifferentiated $\mathrm{SpA}$ (Student-t test for continuous variables and Pearson Chi-square test for categorical variables)

Conclusions: Compared with patients traditionally classified as uSpA, patients who are currently classified as nr-axSpA are diagnosed earlier, are more frequently HLA-B27 carriers and have higher disease activity according to objective parameters. On the other hand, they report lower values for patient reported outcomes.

Disclosure of Interest: None declared

DOI: 10.1136/annrheumdis-2017-eular.5675

\section{SAT0386 EXTREME PATIENT REPORTED OUTCOME (PRO) IN EARLY SPONDYLOARTHRITIS: A SURROGATE FOR FIBROMYALGIA? ITS IMPACT ON TNF-ALPHA BLOCKERS TREATMENT EFFECT?}

B. Meghnathi ${ }^{1}$, A. Etcheto ${ }^{1}$, P. Claudepierre ${ }^{2}$, M. Dougados ${ }^{1}$, A. Moltó ${ }^{1}$. ${ }^{1}$ Paris Descartes University, Medicine Faculty; APHP, Rheumatology B Department, Cochin Hospital; ${ }^{2}$ Paris XII University; Rheumatology Department, Hôpital Henri-Mondor, Paris, France

Background: In case of a concomitant fibromyalgia (FM) with axial spondyloarthritis (axSpA), there is a risk of misclassifying a patient as active (e.g. BASDAI >4), and to falsely consider him as refractory to NSAIDs/ biologics, since FM patients often report higher level of pain and fatigue ${ }^{1}$. We hypothesized that not only are extreme patient reported outcome (PRO) potentially surrogate marker of FM in axSpA, but also that this extreme PRO may have an impact on the TNF- $\alpha$ blockers (TNFb) treatment effect

Objectives: A) To describe the prevalence of extreme PRO in an early axSpA cohort. B) To compare the phenotype of axSpA patients with and without extreme PRO. C) To assess the impact of extreme PRO on the TNFb efficacy.

Methods: This analysis was performed on the DESIR cohort which included 708 adult patients ( $>18$ and $<50$ years) with inflammatory back pain suggestive of axSpA (according to the rheumatologist's conviction of $\geq 5 / 10$ ) for $>3$ months but $<3$ years duration. All patients were biologic naïve at inclusion and were followed up every 6 months for the first 2 years. At baseline, data pertaining to demographics, BASDAl, history of depression, type of medications (antidepressants or muscle relaxants) used and concomitant diseases were collected. It is worth noting that no systematic assessment of the fulfilment of the ACR criteria for FM was performed in this study; thus, we created a "FM gold standard" according to the available data (i.e., presence of either Muscle relaxants/ Depression/ Anti-depressant drug treatment/ Fibromyalgia comorbidity reported in the CRF). BASDAI was the selected PRO for this study due to its widespread use in clinical practice. BASDAI was tested against this "FM gold standard": we plotted ROC curves to define the best cut-off to define an "extreme PRO" for BASDAI. Phenotype of patient's with/without extreme PRO scores was compared. Impact of extreme PRO score on TNFb efficacy was assessed by comparing the retention rate of the first TNFb by Cox analysis.

Results: ROC curves to define an "extreme PRO" determined a different cut-off for each BASDAl question (i.e. $>6,>5,>1,>4,>5$ and $>3$ for question 1 to 6 , respectively), and the need of at least 4 out of these 6 cut-offs to fulfil the "extreme PRO" condition; giving us a prevalence of $42.9 \%$ (304 patients) of extreme PRO in DESIR. Phenotypically, this group with extreme PRO, consisted of older patients (34.6 (8.3) vs $33.1(8.8)$ ), had more females $(184(60.5 \%)$ vs $195(48.8 \%))$, reported less sacroilitis [radiographic and MRI, (36 (12\%) vs 76 $(19.4 \%))$ and $(82(27.6 \%)$ vs $148(37.8 \%))$, respectively], showed less HLA B27 positivity $(160(52.6 \%)$ vs $248(62.2 \%))$, had higher CRP values $(102(34.3 \%)$ vs $102(26.5 \%))$, and more arthritis/ enthesitis history $(212(69.7 \%)$ vs $190(47.5 \%) /$ $182(59.9 \%)$ vs $166(41.5 \%))$. A lower retention rate was observed in the group of patients with "extreme PRO" (Figure 1)

Conclusions: Coexistence of extreme PRO might be considered as a surrogate marker for FM in axSpA patients. Moreover, it appears to have a negative impact on TNF- $\alpha$ blockers retention rate.

\section{References:}

[1] Wendling D, Prati C. Spondyloarthritis and fibromyalgia: interfering association or differential diagnosis? Clin Rheumatol 2016;35:2141-43. 